\title{
Cambios en la distribución etaria de las madres en Chile y en el Hospital Clínico de la Universidad de Chile y su influencia en la morbimortalidad neonatal
}

\author{
Constanza Ramírez R. a, Julio Nazer H. ${ }^{1}$, Lucía Cifuentes 0. ${ }^{2}$, Alfredo Águila R. ${ }^{1}$, \\ Rodrigo Gutiérrez R. a \\ ${ }^{1}$ Unidad de Neonatología, Departamento de Obstetricia y Ginecología, Hospital Clínico Universidad de Chile. ${ }^{2}$ Instituto \\ de Ciencias Biomédicas, Facultad de Medicina, Universidad de Chile.
}

a Alumnos, Escuela de Medicina, Universidad de Chile.

\section{RESUMEN}

Antecedentes: En las últimas décadas en Chile y otros países Sudamericanos se ha producido un cambio en la distribución etaria de las madres. Objetivos: Obtener la distribución de los nacimientos por grupos de edades maternas en el Hospital Clínico de la Universidad de Chile ( $\mathrm{HCUCH})$ y compararlos con iguales grupos en todo Chile. Comprobar que los grupos de edades extremas están aumentando. Estudiar la relación entre estos cambios con algunas patologías del recién nacido, como prematuridad, recién nacidos pequeños para su edad gestacional (PEG) y malformaciones congénitas. Método: Datos de la maternidad del HCUCH (1982-2010) y del Ministerio de Salud (1990-2008). Se distribuye a las madres en 3 grupos: $<20, \geq 35$ y 20-34 años. Las comparaciones se hicieron con prueba de Chi Cuadrado. Resultados: En Chile el grupo de madres $\geq 35$ años aumentó en más del $50 \%$ al comparar los años 1990 (10,2\% del total) y $2008(15,8 \%)\left(p<10^{-8}\right)$. Las madres adolescentes en Chile aumentaron de $13,8 \%$ en 1990 a $16,6 \%$ en $1998\left(p<10^{-8}\right)$. En el HCUCH las adolescentes disminuyeron de $16,08 \%$ en 1982 a $9,09 \%$ en $2010\left(p<10^{-8}\right)$. Las $\geq 35$ años aumentaron significativamente, al igual que en Chile, de $9,8 \%$ en 1982 a $22,17 \%$ en el año $2010\left(p<10^{-8}\right)$. Esta distribución etaria en el HCUCH (1996-2010) se asoció con otros cambios significativos: disminuyeron los RN PEG $(p=0,02)$ y los prematuros $(<37$ semanas) aumentaron significativamente $(p<0,00001)$. El grupo de mujeres $\geq 35$ años se asocia con mayor cantidad de RN femeninos, malformaciones congénitas, síndrome de Down y partos por cesárea.

\section{PALABRAS CLAVES: Edad materna, prematuridad, malformaciones congénitas}

\section{SUMMARY}

Introduction: In recent decades, both in Chile and other South American countries there has been a change in the age distribution of mothers. Objectives: To obtain the distribution of births by maternal age groups in the Hospital Clinic of the University of Chile $(\mathrm{HCUCH})$ and compare them with similar groups throughout Chile. Check that the extreme age groups are increasing. To study the relationship between these changes and some diseases of the newborn, such as prematurity, infants small for gestational age (SGA) and birth defects. Methods: We reviewed the database of the Maternity HCUCH (1982-2010) and of the Ministry of Health (19902008). The mothers are distributed in 3 groups: $<20, \geq 35$ and between $20-34$ years. Comparisons were made with chi-square test. Results: In Chile, the group of mothers $\geq 35$ years old has increased by over $50 \%$ when comparing the $1990\left(10.2 \%\right.$ of total) and $200815.8 \%\left(p<10^{-8}\right)$. Teenager mothers in Chile increased from $13.8 \%$ in 1990 to $16.6 \%$ in $1998\left(\mathrm{p}<10^{-8}\right)$. In the $\mathrm{HCUCH}$ variations were different, adolescents decreased 
$16.08 \%$ in 1982 to $9.09 \%$ in $2010\left(p<10^{-8}\right)$. The $\geq 35$ years increased significantly, as in all of Chile, $9.8 \%$ in 1982 to $22.17 \%$ in $2010\left(p<10^{-8}\right)$. The live birth age distribution in the HCUCH $(1996-2010)$ led to significant changes: reducing SGA $(p=0.02)$, the premature $(<37$ weeks) increased significantly $(p<0.00001)$. The group of women $\geq 35$ years is associated with increased number of children women, congenital malformations, Down syndrome and cesarean deliveries.

\section{KEY WORDS: Maternal age, prematurity, congenital malformations}

\section{INTRODUCCIÓN}

El objetivo principal del ECLAMC (Estudio Colaborativo Latino Americano de Malformaciones Congénitas), del que el Hospital Clínico de la Universidad de Chile es miembro desde 1969, es monitorear las prevalencias al nacimiento de todas las malformaciones congénitas (MFC) e identificar factores de riesgo para realizar prevención primaria de ellas. Uno de los factores de riesgo más importantes que hemos logrado identificar en nuestras publicaciones es la edad materna $(1,2,3,4)$.

Hemos comunicado que el promedio de edad materna de las madres de los recién nacidos en la maternidad del Hospital Clínico de la Universidad de Chile $(\mathrm{HCUCH})$ ha ido aumentando progresivamente, desde 25 años en 1972 a 29,3 años en 2009 (2). Este "envejecimiento" de las madres es un hecho que ha ocurrido en Chile y en muchos países desarrollados (5). En Chile, los nacimientos en mujeres de 40 o más años han aumentado desde 5.773 nacimientos en el año 1990 (18,7/1.000 nacidos vivos) hasta 7.001 (26,5/1.000 nacidos vivos) en 1999. La tendencia actual de las mujeres de postergar la maternidad es un hecho demostrado que se debe a varios factores culturales de la sociedad moderna.

Se ha demostrado la asociación de las edades maternas extremas con malformaciones congénitas $(6,7)$. Las edades maternas avanzadas se relacionan especialmente con malformaciones de origen cromosómico producidas por no disyunción, entre las que se destacan las trisomías 13, 18 y 21 $(8,9,10,11)$. Pero también se ha comunicado mayor riesgo para defectos de tubo neural, especialmente anencefalia y espina bífida (12). Las edades maternas menores, adolescentes, se han relacionado con malformaciones no cromosómicas, de origen disruptivo, como gastrosquisis $(13,14,15)$ y de otro tipo como estenosis pilórica, hidrocefalia, polidactilia, persistencia del ductus arterioso $(15,16)$ y displasia septo-óptica $(14,17,18)$. Se ha especulado sobre los factores que pudieran influir en este grupo etario y se ha mencionado a la alimentación, el consumo de tabaco, el alcohol y las drogas $(19,20)$.

También se ha documentado la asociación de factores como edades maternas extremas con prematu- ridad y restricción del crecimiento intrauterino. Baird y cols (17) y Fraser y cols (18), encontraron que estos factores de riesgo pueden encontrarse en mujeres jóvenes, casadas, con buen nivel socio-cultural y con adecuado control prenatal, por lo que sugieren que la edad materna baja es por sí solo un factor que aumentaría el riesgo adverso del embarazo.

Como grupo de trabajo tenemos la hipótesis de que los grupos de edades maternas extremas (menores de 20 y mayores de 35 años) han ido aumentando en desmedro del grupo intermedio (20-34 años), sin embargo esto aún no ha sido analizado en nuestras publicaciones anteriores.

Los objetivos de este estudio son: 1. Analizar la distribución de tres grupos de edades maternas: menores de 20 años, 20 a 34 años y mayor a 35 años en el Hospital Clínico de la Universidad de Chile HCUCH, durante los años 1982-2010. 2. Analizar y comparar la distribución de tres grupos de edades maternas: menores de 20 años, 20 a 34 años y mayor a 35 años con datos nacionales del MINSAL durante los años 1990-2010. 3. Comparar la distribución etaria de las madres de Chile con la de otros países latinoamericanos. 4. Estudiar la relación entre edad materna con patologías del recién nacido (RN) como anomalías congénitas, prematuridad, pequeños para la edad gestacional y aberraciones cromosómicas.

\section{PACIENTES Y MÉTODO}

Se revisan las estadísticas publicadas por el Ministerio de Salud de Chile desde 1990 y de la Maternidad del HCUCH desde 1982 a 2010. Las madres se distribuyeron en 3 grupos: $<20$ años, 2034 años $y \geq 35$ años. Se comparan los porcentajes de cada grupo de edad materna entre países y a través de los años.

Se buscó la asociación entre grupos de edad materna y sexo del recién nacido $(R N)$, pequeño para la edad gestacional (PEG), prematurez, porcentaje de cesáreas, mortalidad neonatal, síndrome de Down y gastrosquisis. Para el análisis de proporciones se efectuó la prueba de Chi cuadrado, considerando como significativo un valor $\mathrm{p}<0,05$. 


\section{RESULTADOS}

La evolución en Chile de los grupos de edades maternas desde 1990 hasta 2008, se presenta en la Tabla I. Se aprecia una tendencia a la disminu- ción de la natalidad que cayó de 292.145 nacimientos en 1990 a 230.352 nacimientos en 2008 lo que representa una disminución significativa de $22 \%$ $(p<0,05)$, para aumentar levemente en los tres años siguientes hasta 246.145 nacimientos.

\section{Tabla I}

DISTRIBUCIÓN DE LOS NACIMIENTOS SEGÚN GRUPOS DE EDADES MATERNAS EN CHILE (1990 - 2008)

\begin{tabular}{|c|c|c|c|c|}
\hline \multirow[b]{2}{*}{ Año } & \multirow[b]{2}{*}{ Total nacimientos } & \multicolumn{3}{|c|}{ Edad materna (años) } \\
\hline & & $<20(\%)$ & 20-34 (\%) & $\geq 35(\%)$ \\
\hline 1990 & 292.145 & $40.285(13,8)$ & $222.569(76,2)$ & $29.291(10,0)$ \\
\hline 1991 & 284.482 & $39.030(13,7)$ & $214.738(75,5)$ & $30.714(10,8)$ \\
\hline 1992 & 279.098 & $38.483(13,8)$ & $209.366(75,0)$ & $31.249(11,2)$ \\
\hline 1993 & 275.916 & $38.555(14,0)$ & $205.578(74,5)$ & $31.912(11,6)$ \\
\hline 1994 & 273.766 & $38.848(14,2)$ & $202.740(74,1)$ & $32.178(11,8)$ \\
\hline 1995 & 265.932 & $38.885(14,6)$ & $194.332(73,4)$ & $32.715(12,3)$ \\
\hline 1996 & 264.793 & $39.701(15,0)$ & $191.255(72,2)$ & $33.837(12,8)$ \\
\hline 1997 & 259.959 & $40.734(15,7)$ & $184.715(71,1)$ & $34.510(13,3)$ \\
\hline 1998 & 257.105 & $41.530(16,3)$ & $180.568(70,2)$ & $35.007(10,2)$ \\
\hline 1999 & 250.674 & $40.439(16,1)$ & $174.514(69,6)$ & $35.721(14,2)$ \\
\hline 2000 & 248.893 & $40.312(16,2)$ & $171.628(69,0)$ & $36.953(14,8)$ \\
\hline 2001 & 246.116 & $39.884(16,2)$ & $168.278(68,4)$ & $37.954(15,4)$ \\
\hline 2002 & 238.981 & $37.618(15,7)$ & $163.764(68,5)$ & $37.599(15,7)$ \\
\hline 2003 & 234.486 & $34.832(14,9)$ & $161.536(68,9)$ & $38.118(16,3)$ \\
\hline 2004 & 230.831 & $34.424(14,9)$ & $158.264(68,7)$ & $37.664(16,3)$ \\
\hline 2005 & 230.831 & $36.069(15,6)$ & $157.290(68,1)$ & $37.472(16,2)$ \\
\hline 2006 & 231.383 & $37.773(16,3)$ & $156.034(67,4)$ & $37.576(16,2)$ \\
\hline 2007 & 240.569 & $39.605(16,5)$ & $162.754(67,7)$ & $38.165(15,7)$ \\
\hline 2008 & 246.581 & $40.927(16,6)$ & $166.644(67,6)$ & $38.977(15,8)$ \\
\hline
\end{tabular}


Se aprecia que el grupo de madres adolescentes, menores de 20 años, aumentó en forma significativa; en 1990 contribuían con el 13,8\% de los nacimientos y en 2008 aumentó a 16,6\% $\left(p<10^{-8}\right)$. El grupo de 20 a 34 años disminuyó de forma significativa desde $76,2 \%$ a $67,6 \% \quad\left(p<10^{-8}\right)$, mientras que el grupo de madres $\geq 35$ años incrementó en más de 50\%. En efecto, en 1990 constituían el $10,02 \%$ del total para aumentar a $15,8 \%$ en 2008 $\left(p<10^{-8}\right)$. Es decir, en el período estudiado aumentaron significativamente la proporción de nacimientos en los grupos de madres adolescentes y de las mayores de 35 años a expensas del grupo intermedio (20 a 34 años) (Tabla II).

Al analizar la distribución etaria en el $\mathrm{HCUCH}$ observamos que el grupo de madres menores de 20 años disminuyó de $16,8 \%$ a $9,09 \%$ entre los años $1982-2010\left(p<10^{-8}\right)$, el grupo de $\geq 35$ años aumentó de $9,8 \%$ a $22,7 \%$ entre los años 1982-2010 $\left(p<10^{-8}\right)$, y el grupo de $20-34$ años disminuyó desde $74,12 \%$ en 1982 a un $68,74 \%$ en $2010\left(p<10^{-8}\right)$.

La distribución etaria en los países Latino Americanos participantes del ECLAMC, durante el período 1998-2005 se observa en la Tabla III. Observamos que los países con predominio de madres menores de 20 años corresponden a Venezuela y Colombia, en los que un cuarto de los nacimientos corresponde a mujeres adolescentes $(25,3 \%$ y $23,3 \%$ respectivamente), lo que resulta muy superior al promedio del ECLAMC (19,7\%), encontrándose Chile por debajo del promedio del ECLAMC (17,5\%).

Los países que tienen la mayor concentración de madres $\geq 35$ años corresponden a Chile $(14,4 \%)$ y Uruguay $(13,6 \%)$, lo que es superior respecto al promedio del ECLAMC $(11,3 \%)$. Venezuela y Colombia tienen a su vez la menor cantidad de madres $\geq 35$ años $(7,8 \%$ y $6,3 \%$ respectivamente).
Tabla II

FRECUENCIA DE DISTRIBUCIÓN DE EDADES MATERNAS POR GRUPOS ETARIOS. HOSPITAL CLÍNICO UNIVERSIDAD DE CHILE

\begin{tabular}{|c|c|c|c|}
\hline Año & $<20$ años & 20-34 años & >34 años \\
\hline 1982 & 16,1 & 74,1 & 9,8 \\
\hline 1983 & 13,6 & 76,9 & 9,48 \\
\hline 1984 & 13,9 & 74,9 & 11,2 \\
\hline 1985 & 12,5 & 77,3 & 10,3 \\
\hline 1986 & 11,9 & 78,5 & 9,6 \\
\hline 1987 & 7,9 & 80,6 & 11,5 \\
\hline 1988 & 7,3 & 80,7 & 12,0 \\
\hline 1989 & 8,1 & 80,6 & 11,3 \\
\hline 1990 & 11,5 & 77,4 & 11,1 \\
\hline 1991 & 12,7 & 76,6 & 10,8 \\
\hline 1992 & 13,2 & 75,9 & 11,2 \\
\hline 1993 & 12,9 & 73,5 & 13,5 \\
\hline 1994 & 11,1 & 75,9 & 13,1 \\
\hline 1995 & 4,1 & 82,1 & 13,8 \\
\hline 1996 & 4,4 & 81,7 & 13,9 \\
\hline 1997 & 5,7 & 79,6 & 14,8 \\
\hline 1998 & 6,2 & 77,9 & 15,8 \\
\hline 1999 & 5,9 & 75,6 & 18,6 \\
\hline 2000 & 5,9 & 74,5 & 19,6 \\
\hline 2001 & 6,1 & 73,5 & 20,4 \\
\hline 2002 & 5,9 & 74,0 & 20,1 \\
\hline 2003 & 4,9 & 72,3 & 22,8 \\
\hline 2004 & 4,6 & 72,1 & 23,3 \\
\hline 2005 & 6,7 & 71,9 & 21,6 \\
\hline 2006 & 8,2 & 69,4 & 22,5 \\
\hline 2007 & 7,6 & 71,9 & 20,4 \\
\hline 2008 & 10,5 & 70,3 & 19,1 \\
\hline 2009 & 8,5 & 69,1 & 22,4 \\
\hline 2010 & 9,1 & 68,7 & 22,2 \\
\hline
\end{tabular}

Tabla III

DISTRIBUCIÓN DE LOS NACIMIENTOS SEGÚN GRUPOS ETARIOS DE SUS MADRES,
POR PAÍS Y TOTAL DEL ECLAMC (1998-2005)

\begin{tabular}{lrrrr}
\hline País & $<20$ años $(\%)$ & $20-34$ años $(\%)$ & $\geq 35$ años (\%) & Total \\
\hline Argentina & $156.856(19,2)$ & $568.721(69,7)$ & $89.877(11,0)$ & 815.454 \\
Bolivia & $19.387(18,1)$ & $75.011(69,1)$ & $12.886(12,0)$ & 107.284 \\
Brasil & $104.946(20,3)$ & $356.295(68,0)$ & $56.601(10,9)$ & 517.842 \\
Colombia & $15.902(23,3)$ & $44.987(66,0)$ & $7.261(6,3)$ & 68.150 \\
Chile & $65.565(17,5)$ & $255.233(68,1)$ & $53.842(14,4)$ & 374.640 \\
Ecuador & $27.302(20,0)$ & $94.897(69,7)$ & $13.948(10,2)$ & 136.147 \\
Paraguay & $5.820(21,7)$ & $17.779(63,3)$ & $3.206(12,0)$ & 26.805 \\
Uruguay & $14.688(15,6)$ & $67.034(71,0)$ & $12.777(13,6)$ & 94.449 \\
Venezuela & $59.652(25,3)$ & $15.803(67,0)$ & $18.509(7,8)$ & 236.193 \\
ECLAMC & $475.711(19,7)$ & $1.660 .620(68,9)$ & $273.076(11,3)$ & 2.409 .407 \\
\hline
\end{tabular}


Tabla IV

CARACTERÍSTICAS DE LOS RECIÉN NACIDOS Y TIPO DE PARTO SEGÚN EDAD MATERNA

\begin{tabular}{cccccccccc}
\hline $\begin{array}{l}\text { Edad } \\
\text { (años) }\end{array}$ & $\mathrm{n}$ & $\begin{array}{c}\% \\
\text { masculino }\end{array}$ & $\begin{array}{c}\% \\
\mathrm{PEG}\end{array}$ & $\begin{array}{c}\% \\
<37 \mathrm{~s}\end{array}$ & $\begin{array}{c}\% \\
\text { fallecidos }\end{array}$ & $\begin{array}{c}\% \\
\text { MFC }\end{array}$ & $\begin{array}{c}\% \\
\text { Cs }\end{array}$ & $\begin{array}{c}\% \\
\text { SD }\end{array}$ & $\begin{array}{c}\% \\
\mathrm{GQ}\end{array}$ \\
\hline$<20$ & 1.791 & 52,1 & 8,6 & 16,2 & 0,67 & 7,4 & 27,0 & 0,06 & 0,11 \\
$20-34$ & 19.901 & 51,3 & 9,7 & 17,5 & 0,75 & 8,5 & 38,5 & 0,13 & 0,06 \\
$\geq 35$ & 5.270 & 49,7 & 10,8 & 23,2 & 0,85 & 10,0 & 0,92 & 0,92 & 0,0 \\
Valor p & - & $<0,049$ & $<0,011$ & $<0,0001$ & 0,65 & $<0,0002$ & $<0,0001$ & $<0,0001$ & 0,11 \\
\hline
\end{tabular}

PEG: pequeño para la edad gestacional. MFC: malformaciones congénitas. Cs: cesáreas. SD: síndrome de Down. GQ: gastrosquisis.

Las patologías del recién nacido respecto a los grupos de edades maternas para el HCUCH durante el período 1996-2010 se observan en la Tabla IV. Observamos que el grupo de madres $\geq 35$ años presentó predominio de hijos femeninos en relación a masculinos $(p<0,049)$. Además este grupo presentó significativamente mayor frecuencia de recién nacidos PEG $(p<0,011)$, más prematurez $(p<0,0001)$, más hijos malformados $(p<0,0002)$, mayor frecuencia recién nacidos portadores de Síndrome de Down $(p<0,0001)$ y más partos por cesárea $(p<0,0001)$. Sin encontrar diferencias significativas en relación a gastrosquisis y mortalidad neonatal.

\section{DISCUSIÓN}

Al analizar la distribución etaria materna tanto a nivel nacional como en el $\mathrm{HCUCH}$, podemos observar que esta ha ido cambiando a lo largo del tiempo. En ambas muestras las madres $\geq 35$ años aumentaron en forma significativa. Sin embargo, mientras que en el total de Chile el grupo de madres $<20$ años ha aumentado significativamente, en el HCUCH disminuyó en forma significativa, debido seguramente, a que la población atendida en este hospital es distinta al resto de Chile. Los datos de Chile son los que proporciona el Ministerio de Salud de sus hospitales públicos y privados a diferencia del $\mathrm{HCUCH}$ que es solamente atención privada. El HCUCH pasó de ser un hospital público a uno privado en 1994 por lo que su distribución etárea actualmente se comporta como los centros privados con mayor predominio de edades mayores. Esta hipótesis, se apoya en un estudio nacional en donde se compara una institución pública con una privada (Hospital San Borja Arriarán y la Clínica Las Condes) con edades promedio del parto para el hospital público de 25,65 $\pm 0,92$ años respecto a la Clínica Las Condes de 32,73 $\pm 0,110$ años $(p<0,05)$ y la proporción de primíparas adolescentes $(<20$ años de edad) en el hospital público correspondió a $42,4 \%$ respecto a la clínica privada con un $1,6 \%$ (28).

Al analizar la distribución de las edades maternas entre los distintos países participantes del ECLAMC observamos gran heterogeneidad entre ellos. Podemos observar que países como Chile y Uruguay presentan una tendencia a comportarse como países más desarrollados, los que habitualmente también presentan postergación de la maternidad. Los porcentajes de edad materna $\geq 35$ años de ambos países (14,4\% y 13,6\%), se asemejan a lo observado el año 2005 en Austria y Dinamarca donde correspondieron $17,5 \%$ y $17,4 \%$ respectivamente (29). En cambio, países como Colombia y Venezuela presentan predominio de madres <20 años $(25,3 \%$ y $23,3 \%)$, lo que resulta hasta 17 veces más respecto a países como Dinamarca 1,3\% y Suiza 1,1\% (30).

Además, en la Tabla III se puede observar un hecho paradojal; se sabe por múltiples publicaciones que la edad materna es el factor de riesgo más importante para síndrome de Down. Según los datos del ECLAMC, Chile que tiene la mayor cantidad de madres $\geq 35$ años, tiene a su vez, como era de esperar, la mayor prevalencia al nacimiento de esta patología en la región (2,47 por mil nacimientos) (2). Sin embargo, Uruguay que presenta la segunda mayor cantidad de madres en el grupo de $\geq 35$ años, presenta la menor prevalencia de síndrome de Down (1,32 por mil), que nos hace pensar que existen otros factores de riesgo, además de la edad materna, que influyen en la frecuencia de esta patología (18).

Al analizar la relación entre los grupos de edades maternas respecto a la frecuencia de patologías del recién nacido (Tabla IV) observamos que hay una relación significativa con patologías como la prematurez, lo que está en concordancia con lo comunicado por otros autores en Perú (21), Chile $(22,23)$ y España $(24)$, que comunican aumento 
de los partos pretérmino en mujeres de más de 34 años.

Respecto al aumento de las cesáreas, esto también concuerda con lo publicado en estudios internacionales y por otro grupo nacional que compara mujeres $\geq 35$ años respecto a las de 20-34 años, con un riesgo significativamente más alto en las mujeres mayores, debido fundamentalmente complicaciones como síndrome hipertensivo del embarazo y diabetes gestacional, entre otros $(8,31,32)$.

Respecto a la asociación entre edad materna y malformaciones congénitas esto se encuentra ampliamente demostrado y ratificado en múltiples publicaciones por lo que solo concuerda con lo publicado por este grupo anteriormente $(2,8)$. Dentro de ellas se encuentran las alteraciones cromosómicas y el síndrome de Down cuya asociación con edades maternas avanzadas ya ha sido publicado por este grupo (1).

Respecto al bajo peso de nacimiento, también nuestros datos concuerdan con otro estudio nacional (22) y otro internacional (24) en donde se comparan las madres $\geq 35$ años respecto a las entre 20-34; las primeras presentan más $\mathrm{RN}$ de bajo peso al nacer, respecto a las más jóvenes.

\section{CONCLUSIÓN}

Tanto en el HCUCH como en todo Chile se produjo un cambio significativo en la distribución etaria de las madres, en ambos las edades maternas $\geq 35$ años han aumentado, y las <20 años aumentaron a nivel nacional y disminuyeron a nivel local. En el $\mathrm{HCUCH}$ el grupo de madres $\geq 35$ años se asocia con significativa mayor frecuencia de prematuros, malformaciones congénitas, PEG, síndrome de Down, partos por cesárea y RN femeninos. Sin diferencias significativas en cuanto a mortalidad neonatal o gastrosquisis.

\section{REFERENCIAS}

1. Nazer J, Cifuentes L. Estudio epidemiológico global del síndrome de Down. Rev Chil Pediatr 2011;82:105-12.

2. Nazer J, Cifuentes L, Aguila A, Ureta P, Bello MP, Correa $F$, Melibosky R. Edad materna y malformaciones congénitas. Un registro de 35 años: 1990-2004. Rev Méd Chile 2007;135:1463-69.

3. Nazer J, Aguila A, Cifuentes L. Vigilancia epidemiológica del síndrome de Down en Chile. 1972-2005. Rev Méd Chile 2006;134: 549-57.

4. Nazer J, Eagling MA, Cifuentes L. Incidencia del Síndrome de Down en la maternidad del Hospital Clínico de la Universidad de Chile. Un registro de 25 años. Rev Méd Chile 1998;126:383-90.

5. Donoso E, Carvajal J, Domínguez MA. Reducción de la fecundidad y envejecimiento de las mujeres chilenas en edad fértil: 1990-2004. Rev Méd Chile 2009;137:766-73.

6. Pardo RA, Nazer J, Cifuentes L. Prevalencia al nacimiento de malformaciones congénitas y de menor peso de nacimiento en hijos de madres adolescentes. Rev Méd Chile 2003; 131: 1165-72.

7. Donoso E, Becker J, Villarroel L. Natalidad y riesgo reproductivo en adolescentes en Chile, 1990-1999. Rev Panam Salud Pública 2003;14:3-8.

8. Chamy V, Cardemil F, Betancour P, Ríos M, Leighton L. Riesgo obstétrico y perinatal en embarazadas mayores de 35 años. Rev Chil Obstet Ginecol 2009;74:331-8.

9. Reefhuis J, Honein M. Maternal age and chromosomal birth defects. Atlanta 1998-2000: teenager or thirty-something, who is the risk? Birth Defects Res A Clin Mol Teratol 2004;70:572-9.

10. Sherman SL, Freeman SB, Allen EG, Lamb NE. Risk factors for nondisjunction of trisomy 21. Citogenetic Genome Res 2005;111:273-80.

11. Cocchi G, Gualdi S, Bower C, Halliday J, Jonsson B, Mastroiacovo $\mathrm{P}$, et al. International trends of Down syndrome 1993-2004: Birth relation to maternal age and terminations of pregnancies. Birth Defects Res A Clin Teratol 2010;88:474-9.

12. Vieira A, Castillo S. Edad materna y defectos de tubo neural: evidencia para un efecto mayor de espina bífida que anencefalia. Rev Méd Chile 2005;133:62-70.

13. Kasaura MR, Lie RT, Irgens LM, Didriksen A, Kapstad $\mathrm{M}$, Egenaes $\mathrm{J}$, et al. Risk of gastroschisis in Norway: An age-period-cohort analysis. Am $\mathrm{J}$ Epidemiol 2004;159:358-63.

14. Lubinsky MS. Association of prenatal vascular disruptions with decreased maternal age. Am J Med Genet 1997;69:237-9.

15. Reefhuis J, Honein M. Maternal age and chromosomal birth defects. Atlanta 1998-2000: teenager or thirty-something, who is the risk? Birth Defects Res A Clin Mol Teratol 2004;70:572-9.

16. Hollier LM, Leveno KJ, Kelly MA, Mclntire DD, Cunningham FG. Maternal age and malformations in singleton births. Obstet Gynecol 2000;96:701-6.

17. Baird PA, Sudovnik AD, Yee IM. Maternal age and birth defects: a population study. Lancet 1991;337:527-30.

18. Fraser AM, Brockert JE, Ward RH. Association of young maternal age with adverse reproductive outcomes. N Engl Med 1995;332:1113-7.

19. Kovaleva N, Mutton D. Epidemiology of double aneuploidies involving chromosome 21 and sex chromosomes. Am J Med Genet A 2005;134 A:24-32.

20. Centers for Disease Control. Congenital Surveillance U.S. Department of Health and Human Service. Teratology 1993;48:545-82.

21. Tipiani-Rodríguez O. ¿Es la edad materna avanzada un factor de riesgo independiente para complicaciones materno-perinatales? Rev Per Ginecol Obstet 2006;52:179-85.

22. Donoso E, Villarroel L. Edad materna avanzada y riesgo reproductivo. Rev Méd Chile 2003;131:55-9.

23. Molina R. Niñas madres en Chile: un problema que sigue sin solución. (Editorial) Rev Chil Obstet Ginecol 2010;75:347-8. 
24. Luque Fernández MA. Evolución del riesgo de mortalidad fetal tardía, prematuridad y bajo peso al nacer, asociado a la edad materna avanzada, en España (1996-2005). Gaceta Sanitaria 2008;22:396-403.

25. Hernández Cabrera J, Alonso Hernández D, Rodríguez Pino M, Homma Castro JL, Díaz Olano I, Suárez Ojeda R. Embarazo en adolescentes y su influencia en los indicadores perinatales en 2 hospitales ginecobstétricos. Rev Cubana Obstet Ginecol 2003;29(3) Disponible en: http://bvs.sld.cu/revistas/gin/vol29_3_03 / gin04303.htm.

26. Instituto Nacional de Estadísticas. Anuario de Demografía. Chile, 1990.

27. Instituto Nacional de Estadísticas. Anuario de Demografía. Chile, 1999.

28. Fuentes A, Jesam C, Devoto L, Angarita B, Galleguillos $A$, et al. Postergación de la maternidad en Chile:
Una realidad oculta. Rev Méd Chile 2010;138:1240-5

29. Percentage of live births to mothers aged 35 and over in Iceland, Norway, Switzerland and the EU-27, 19902005. EUPHIX: European Union Public Health Information.

30. Percentage of live births to mothers aged under 20 in Iceland, Norway, Switzerland and the EU-27, 19902005. EUPHIX: European Union Public Health Information.

31. Bayrampour H, Heaman M. Advanced maternal age and the risk of cesarean birth: A systematic review. Birth 2010;37:219-26.

32. Heras Pérez B, Gobernado Tejedor J, Mora Cepeda P, Almaraz Gómez A. La edad materna como factor de riesgo obstétrico. Resultados perinatales en gestantes de edad avanzada. Prog Obstet Ginecol 2011. doi:10.1016/j.pog.2011.06.012. 